\title{
A New Therapeutic Strategy and Rehabilitation in Hereditary Collagen Diseases: Case Report
}

\author{
Angelo P. Amico*, Ilaria Covelli, Marisa Megna and Pietro Fiore \\ Department of Physical Medicine and Rehabilitation, Policlinico of Bari, University of Bari, Bari, \\ Italy
}

\begin{abstract}
The Ehlers-Danlos syndrome (EDS) is the most common hereditary disorder of connective tissue, with main manifestations in skin, joints and cardiovascular system. Hyperelasticity of skin, joint hypermobility and fragility of vessels and internal organs are responsible for severe physical disability and loss of autonomy in ADL (activities of daily life). Aim of the Study: To demonstrate efficacy in patients with hereditary disorders of connective tissue of dietary supplements based on collagen hydrolyzate in association with proprioceptive rehabilitation, by Prokin system, in improving postural stability, osteo-articular painful symptoms and bone density for the recovery of autonomy in ADL. Materi-

Angelo P. Amico als and Methods: A patient of 53 years with genetic diagnosis of Ehlers-Danlos Syndrome type Classic (mutation COL1A1) who had several sprains in the lower limbs, complained pain syndrome in the left ankle and right knee, associated with postural instability. We assessed him by WOMAC, VAS pain scale and Tinetti Balance Scale; by proprioceptive multiaxial test of Prokin; and by bone densitometry. Then he underwent 2 cycles of 10 sessions of proprioceptive rehabilitation with Prokin. In addition, he was prescribed therapy for one month with the PROTOCOL dietary supplement of collagen hydrolyzate $1 \mathrm{flc} /$ day. At the end of the 2 cycles, all the previous tests and evaluations were repeated. Results: The comparison between the scores at T0 (one day before treatment) and T1 (one day after treatment) showed a reduction of pain and stiffness and an improvement of physical function (WOMAC scale from 26/96 to 0/96, VAS of pain from "intense" to "no pain". Postural instability measured by Tinetti balance scale reduced at time $\mathrm{t} 1$ compared to t0; This finding has been confirmed by proprioceptive test of prokin, with a reduction in the percentage of error (ATE) from $34 \%$ to $20 \%$, and the index of instability from 1.2 to 0.80 . After a month of therapy PROTOCOL, dietary supplement of hydrolyzed collagen $1 \mathrm{flc} /$ day, a reduction in the T-score at DEXA from -2.6 to -1.6 was documented. Also at clinical examination at $\mathrm{T} 1$, we found reduced postural instability in monopodalic standing, and less critical postural changes, with significant improvement of functional independence and quality of life of the patient. Conclusions: The preliminary results show how the integration of a proprioceptive rehabilitation, such as Prokin exercises, with dietary supplementation of collagen can lead to a reduction (in this case a resolution) in pain and a functional improvement and recovery of autonomy in ADL.
\end{abstract}

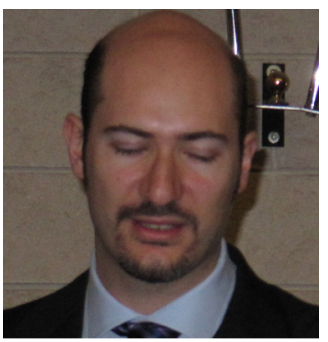

Keywords: Collagen supplements, Ehlers-Danlos syndrome, hereditary disorders, Prokin, rehabilitation, Tinetti Balance Scale.

\section{INTRODUCTION}

The Ehlers-Danlos syndrome (EDS) is the most common hereditary disorder of connective tissue [1], with a maximum manifestation in the soft tissues and, in particular, skin, joints and cardiovascular system. Hyperelasticity of skin, joint hypermobility and fragility of vessels and inner organs are the most representative clinical signs of EDS. EDS presents often a systemic involvement, but in most cases, joint laxity is responsible for severe physical disability and, to a lesser extent, complications of cardiovascular origin. The heterogeneous molecular and biochemical background leads to various degrees of involvement of tissues and organs that characterize the different forms of EDS [2].

The estimated prevalence for all the various forms of EDS varies between $1 / 10000$ and $1 / 25000$ [3] but it is

\footnotetext{
*Address correspondence to this author at the Department of Physical Medicine and Rehabilitation, Policlinico of Bari, University of Bari, Bari, Italy; Tel: 0039 3490576970; E-mail: paolo-amico@alice.it
}

believed that because of its difficult clinical diagnosis, very often they are not recognized and diagnosed, so this frequency would be underestimated.

The various disorders of the EDS are classified according to the involvement of joints and skin [4] Individuals with classical form (EDS I / II) have increased extensibility of the skin, impaired wound healing, atrophic scars, frequent bruising, joint laxity, and recurrent dislocations [5].

The mucocutaneous involvement has certainly been one of the main elements that provoked the first interest in EDS. In these conditions, the skin is usually soft, velvety and hyperelastic. Joint hypermobility is definitely the most characteristic phenotypic part, though it does not specify the type of EDS.

Joint hypermobility is evaluable by measuring the range of motion (ROM) of joints of interest. In the EDS, joint hypermobility is often congenital (and therefore not associated with trauma or overuse) and generalized, although in some 
forms, it is more (or exclusively) evident in the extremities, as in vascular form. For each joint there are reference values of ROM for adulthood and their excursion is usually measured with orthopedic protractors and / or flexible meters.

Neuromuscular aspects of EDS, currently considered relevant in clinical practice, were for decades neglected by the international literature and, consequently, from textbooks. Only recently, right emphasis has been placed on them. The muscular hypotonia, usually not severe, is a common sign and its likely congenital (or prenatal) origin can be responsible, at least partly, for some orthopaedic dismorphisms of the EDS as flat foot, valgism of toes, knees and elbows, mild scoliosis, arched palate and plagiocephaly. In later phases, you may find a loss of strength in various muscle groups and / or muscle wasting, usually in the thenar and hypothenar eminences and interosseous muscles. Myogenic articular retractions, as camptodactylism and clubfoot, are possible, although rare, and often preferentially associated with some forms, such as vascular form. The most important neurological sign is the proprioceptive deficit [6], which is expressed by a positive sign of Romberg (instability in the upright more evident with their eyes closed) in the absence of other signs of vestibular dysfunction. Tactile sensitivity is usually harmless although some patients report hypoesthesia areas (not only in the extremities) or significant but nonspecific differences of tactile sensation between the two halves of the body. Sometimes, a four limbs electromyography maybe performed for differential diagnosis or subclass of the neuromuscular phenotype. Very frequent symptoms from the musculoskeletal system involvement are: sprains and recurrent dislocations slow healing of joint traumas, tendonitis, tenosynovitis, synovitis and recurrent, chronicrelapsing joint pain, muscular soreness, muscle lesions and tendon ruptures; such injuries are often responsible for the worsening of the quality of life of the patients with EhlersDanlos syndrome and therefore are of great rehabilitative interest. To date, there are no specific treatments for this disease. New proposals are based on growing scientific evidence that nutritional deficiencies are important factors in the pathogenesis of many diseases, which were once thought to come only from genetic defects. It was also recognized that many of the symptoms associated with Ehlers-Danlos syndrome are also characteristic of nutritional deficiencies; therefore, the synergistic action of appropriate combinations of food supplements within the body can be useful to promote the formation and function of normal tissue [7].

\section{MATERIALS AND METHODS}

A patient of 53 years with genetic diagnosis of EhlersDanlos Syndrome, type Classic, with a heterozygous dominant mutation in the gene COL1A1, c.934C > t (p.R312C), came to our attention in the Department of Physical and rehabilitative Medicine of Policlinico of Bari on January 2014. The patient reported multiple and relapsing joint sprains in the lower limbs and complained pain in the left ankle and in the right knee, associated with postural instability, which in September 2011 had led him to an accidental fall and consequent injury of the anterior cruciate ligament and right tibial plateau fracture with worsening of pain symptoms and limitation in ADL (activities of daily life) and postural transfers, especially going down the stairs. MRI of December 2011 showed: "lesion in anterior cruciate ligament and in the body-posterior horn of the medial meniscus in an area of degenerative meniscopathy. Abundant joint effusion; gonarthrosis; patellofemoral chondromalacia and bone marrow oedema of the spongy mass in the posterior median paramedian external tibia, were reported as a result of recent bone injury".

In 2012, for the presence of bright red blood in the stool, he underwent colonoscopy that concluded for internal hemorrhoids. In echocardiogram of April 2013, mesotelesistolic mild mitral regurgitation, minimal aortic regurgitation, LV increased in volume. Mitral valve prolapses with mild regurgitation and aortic root dilatation were observed. In July 2013, he performed CT angiography of aorta and epiaortic vessels with evidence of "broncopneumopathic chronic emphysema, paraseptal bubbles, and apical subpleuric fibrotic striae in the right side. Cysts of the liver segment VII and right kidney".

\section{Previous Diagnosis of Osteoporosis}

General physical examination: Normotype with fair overall clinical condition. Skin: shiny and thin, with areas of dischromia in the lower limbs; normal heart area; Heart sounds: two valid and rhythmic; Heart rate: $70 \mathrm{bpm}$; Blood pressure: 130/85 mmHg; Pulses: evaluable and free of murmurs; Chest of frusto-conical shape; normally expanded and expandable chest walls; clear sound at lung percussion; Vescicular murmur spreading widely over the lung. Abdomen: plano-convex, treatable, and painless to superficial and deep palpation; liver and spleen of normal size; Percussion: widespread tympanism; Auscultation: widespread borborygmi.

\section{At the Neurologic and Physiatric Exam}

Well oriented in space and time, and auto- and allopsychically; standing up independently; Postural instability in monopodalic standing; Postural changes possible with adaptations; Independent walking for short to medium routes, with escape lameness, more obvious on the right; Joint ROM increased slightly in the extension of the forearm bilaterally ( $5^{\circ}$ more); Moderate varus knee, intercondylar distance of 5 $\mathrm{cm}$; Presence of multiple bruises on both knees; Thin, hyperextensible skin and telangiectasias; Pain at a pressure of pes anserinus in his right knee; and Flat foot with hallux valgus bilaterally. We administered to the patient the WOMAC scale (n. 24 items, with a maximum score of 96, it investigates pain, stiffness and physical function), VAS pain scale (visual analogue scale of pain with two ends that correspond to a "no pain", other "maximum pain", giving a value from 0 to 10) and Tinetti Balance Scale (n. 25 items, divided into two parts with maximum score of 32 , it investigates balance and gait), and we performed a multiaxial proprioceptive test by the balance platform "Prokin" (which detects the instability index and the average percentage of "errors", index ATE Average Track Error, while the patient describes a circular trace on the screen by performing a circular motion with his ankle). He was recommended to perform bone densitometry and treatment of proprioceptive rehabilitation with platform Prokin (10 sessions with right leg and 10 sessions with left leg). 
At the end of the first cycle of 10 sessions of proprioceptive rehabilitation with platform Prokin, we repeated proprioceptive multiaxial assessment for each foot.

At the end of the second cycle (other 10 sessions) of proprioceptive rehabilitation with platform Prokin, we again administered WOMAC, VAS and Tinetti scales and repeated proprioceptive multiaxial assessment. We prescribed oral therapy for a month with PROTOCOL, a dietary supplement based on collagen hydrolyzate $1 \mathrm{flc} /$ day. After this therapy, we again administered WOMAC, VAS and Tinetti scales and repeated bone densitometry.

\section{PATIENT CONSENT}

A written patient consent was obtained for all diagnostic and therapeutic procedures.

\section{RESULTS}

We compared the results obtained from the WOMAC, VAS and Tinetti scales, the multiaxial proprioceptive assessment of platform prokin, the physiatric clinical examination and DEXA bone densitometry at time t0, i.e. before starting treatment with rehabilitation proprioceptive footboard Prokin and before the treatment with the PROTOCOL supplement, and at time $\mathrm{t} 1$ that is the end of treatments; the results are summarized in Table $\mathbf{1}$.

Table 1. Comparison of results at time to and the time t1.

\begin{tabular}{|c|c|c|}
\hline $\begin{array}{c}\text { Clinical and instrumental } \\
\text { scores }\end{array}$ & T0 & T1 \\
\hline \hline WOMAC scale & $26 / 96$ & $0 / 96$ \\
\hline VAS scale & Intense pain & No pain \\
\hline Tinetti scale & $19 / 32$ & $24 / 32$ \\
\hline $\begin{array}{c}\text { Multiaxial proprioceptive } \\
\text { rating }\end{array}$ & $\begin{array}{c}\text { ATE }=34 \% \\
\text { Instability index } \\
=1,20\end{array}$ & $\begin{array}{c}\text { ATE }=20 \% \\
\text { Instability index } \\
=0,80\end{array}$ \\
\hline DEXA & T SCORE $=-2,6$ & T SCORE $=-1,6$ \\
\hline
\end{tabular}

The comparison showed an improvement between the time t0 with respect to time $\mathrm{t} 1$ of pain, stiffness and physical function, expressed by the improvement of WOMAC scale from 26/96 to $0 / 96$, as well as the visual analogue scale of pain from "pain intense "to" no pain ". The postural instability measured by Tinetti balance scale is reduced at the time tl compared to the time $t 0$; this finding has been confirmed through the multiaxial proprioceptive evaluation implemented by Prokin system, and in particular, a reduction is documented in the percentage of error (ATE) from 34\% to $20 \%$, and the index of instability from 1.2 to 0,80 .
After a month of therapy with PROTOCOL, dietary supplement of hydrolyzed collagen, 1 flc / day, we documented a reduction of the T-score of the DEXA from -2.6 to -1.6.

Also comparing physiatric examination at $\mathrm{t} 1$ with $\mathrm{t} 0$, with reduced postural instability in monopodalic standing, the postural changes appeared less critical. At the same time, we had an improvement of the gait pattern, with a great reduction of escape lameness, and the absence of elicitable pain on the area of pes anserinus in his right knee, with significant improvement of functional independence, of postural changes and of the quality of life of the patient.

\section{CONCLUSION}

Ehlers-Danlos syndrome is responsible for severe disability; besides, phenotypic heterogeneity and the lack of knowledge on the pathogenesis of various clinical manifestations make it often very difficult to diagnose and even more to treat short term and long term symptoms. With this work, we have shown how the integration of the classical rehabilitative therapy, which can use technological systems such as the Prokin, with dietary supplementation of collagen, could be able to lead to an improvement and in this case, the resolution of pain, with great benefit in the recovery of autonomy in ADL.

Surely our purpose for the future is to check this treatment also in other patients with Ehlers Danlos or other collagenopathies, and try to distinguish better the effect of instrumental proprioceptive rehabilitation and the one of collagen supplements.

\section{CONFLICT OF INTEREST}

The authors confirm that this article content has no conflict of interest.

\section{ACKNOWLEDGEMENTS}

Declared none.

\section{REFERENCES}

[1] Rand-Hendriksen S, Wekre LL, Tidsskr PB. Ehlers-Danlos syndrome--diagnosis and subclassification. Tidsskr Nor Laegeforen 2006; 126(15): 1903-7.

[2] De Paepe A, Malfait F. The Ehlers-Danlos syndrome, a disorder with many faces. Clin Genet 2012; 82(1): 1-11.

[3] Germain DP. Ehlers-Danlos syndrome type IV. Orphanet J Rare Dis 2007; 2: 32 .

[4] Malfait F, Wenstrup RJ, De Paepe A. Clinical and genetic aspects of Ehlers-Danlos syndrome, classic type. Genet Med 2010; 12(10): 597-605.

[5] Byers PH, Murray ML. Heritable collagen disorders: the paradigm of the Ehlers-Danlos syndrome. J Invest Dermatol 2012; 132 (E1) E6-11.

[6] Lee HM, Cheng CK, Liau JJ. Correlation between proprioception, muscle strength, knee laxity, and dynamic standing balance in patients with chronic anterior cruciate ligament deficiency. Knee 2009; 16(5): 387-91.

[7] Mantle D, Wilkins RM, Preedy V. A novel therapeutic strategy for Ehlers-Danlos syndrome baEDS on nutritional supplements. Med Hypotheses 2005; 64(2): 279-83. 